\title{
Hand-opening feedback for myoelectric forearm prostheses: Performance in virtual grasping tasks influenced by different levels of distraction
}

\author{
Heidi J. B. Witteveen, MSc; ${ }^{*}$ Leonie de Rond, BSc; ${ }^{1}$ Johan S. Rietman, PhD, MD; ${ }^{2}$ Peter H. Veltink, PhD ${ }^{1}$ \\ ${ }^{1}$ Department of Biomedical Signals and Systems, University of Twente, Enschede, the Netherlands; ${ }^{2}$ Roessingh \\ Research and Development, Enschede, the Netherlands
}

\begin{abstract}
Sensory feedback and the required attentional demands are important aspects in prosthesis acceptance. In this study, hand-opening feedback is provided and the performance in a virtual grasping task is investigated. Simultaneously, a secondary task was performed to investigate the attentional demands. Ten nondisabled subjects performed the tasks with and without feedback about the hand opening through an array of eight vibrotactile stimulators on the forearm. Activation of one stimulator corresponded to one hand-opening position. For the dual-task experiments, subjects simultaneously performed a secondary auditory counting task. The addition of vibrotactile feedback increased the performance (expressed in percentages of correct hand positions, mean absolute errors in position, and percentages of deviations up to one hand-opening position), but the duration of the tasks was also increased. Three levels of distraction (no distraction, counting task, count and subtract task) were applied, which did not influence the performance in the grasping tasks except for the highest level of distraction. We concluded that the proposed method to provide hand-opening feedback through an array of eight vibrotactile stimulators is successful because the performance in a grasping task increases but it is not significantly attention demanding.
\end{abstract}

Key words: attentional demands, auditory tasks, grasping performance, grasping task, hand opening, hand-opening feedback, myoelectric forearm prosthesis, secondary tasks, vibrotactile stimulation, virtual setup.

\section{INTRODUCTION}

The introduction of myoelectric prostheses has led to the development of prostheses with higher levels of functionality. However, these kind of prostheses lack the ability to provide the prosthesis user with adequate sensory information [1]. In contrast with body-powered prostheses, where the control cables can provide force and some position feedback [2], the visual and, to some extent, auditory sensory systems are the only systems that provide some sensory awareness in myoelectric prostheses. This causes a high mental burden on the user to continuously use his or her visual sensory system to control the prosthesis [3]. Pons et al. showed that because of the limited controllability and burden on the visual system required, 30 to 50 percent of the prostheses are not used on a regular basis [4]. Adding sensory feedback, and thereby reducing required visual attention, has been indicated to be an

\footnotetext{
Abbreviations: ANOVA = analysis of variance, VAS = visual analog scale.

*Address all correspondence to Heidi J. B. Witteveen, MSc; Department of Biomedical Signals and Systems, University of Twente, Drienerlolaan 5, Enschede 7522 NB, the Netherlands; +31-53-489-2766; fax: +31-53-489-2287.

Email: h.j.b.witteveen@ewi.utwente.nl

http://dx.doi.org/10.1682/JRRD.2011.12.0243
} 
important improvement in upper-limb prostheses to increase acceptability [4-5]. Furthermore, Peerdeman et al. concluded from a workshop with representative prosthesis users that feedback should be added to reduce the attentional demands and allow intuitive grasping [6]. Feedback about the grasping force and the hand opening were shown to be especially important and should be incorporated in future upper-limb prostheses.

Only a few studies have investigated the use of position feedback in upper-limb prostheses. In an early approach by Mann and Reimers, vibrotactile stimulation was used to provide feedback about the elbow angle [7]. They placed two stimulators on the upper arm and activated them simultaneously with differing amplitudes. The proportion of the amplitudes determined the location in between the stimulators where the stimulation was perceived (concept of phantom sensations). Although their results were promising, no applications of this method can be found. Furthermore, large vibrators and a stabilization plate were necessary to create the sensations [8]. Dhillon and Horch undertook another approach to provide feedback about the elbow angle [9]. They used implanted electrodes to stimulate the individual afferent nerves.

Prior and Lyman incorporated hand-opening feedback using one single electrode to provide feedback about the gripping force by pulse width modulation and about the hand opening by pulse rate modulation [10]. Although they showed that hand-opening feedback increases the ability to distinguish object sizes, no applications are found in current prostheses. One reason for this could be the small range between sensation and pain thresholds with electrotactile stimulation, which results in small stimulation ranges [11-12].

Recent approaches have focused on proprioceptive feedback about the movement of the index finger in a grasping movement [13-14]. A virtual index finger was controlled by force input from the thumb while feedback about the position of the index finger was provided by moving the real index finger with a motor. Although not directly applicable in forearm prostheses, these studies show that proprioceptive feedback increases the awareness of finger movements.

Not only the lack of feedback can lead to the abandonment of the myoelectric prosthesis, but also the required attention and concentration needed to control the prosthesis [15]. As stated by Cipriani et al., "Acceptability is more dependent on the required attention than on the success in grasping” [16]. The addition of feedback creates an extra level of attention required to control the prosthesis, which should be kept as low as possible. In the ideal situation, the feedback can be used subconsciously without requiring extra attention, and extra external distraction would not significantly influence the performance with the prosthesis. None of the previously mentioned studies on position feedback have investigated the attentional demands of the feedback. A way to investigate these attentional demands is using dual-task methodology [17-18]. It is assumed that there is a fixed processing capacity for each individual [19], which is (partly) filled when feedback is used to control the hand opening of a prosthesis. The capacity used determines the attentional demand of the task. The addition of a secondary task requires extra processing capacity to perform both tasks. A high attention-demanding primary task will require a large part of the processing capacity, thereby reducing the available capacity for the secondary task. Therefore, the performance of the secondary task is an indicator of the attentional demand of the primary task. A restriction for these experiments is that the performance of the primary task should be kept constant, which is not always possible. To solve this, researchers evaluated the performance of the primary task while keeping the performance of the secondary task constant. Stepp and Matsuoka showed an example of this approach [20]. They evaluated the effect of distraction on an object displacement task in which feedback was provided about the force that was applied by the subject. The secondary task they used was an auditory calculation task. Their results showed a decrease in speed and an increase in difficulty but no effect of distraction on their main outcome parameter for the performance of the primary task.

In this study, we placed an array of eight vibrotactile stimulators on the forearm to provide feedback about the hand opening of a prosthesis. Our aim was to evaluate the improvements in performance in a grasping task that could be achieved by this feedback method and its attentional demands. We investigated the latter using dual-task methodology.

\section{METHODS}

\section{Subjects}

All experiments were performed with 10 nondisabled subjects ( 6 male and 4 female; age $25.2 \pm 2.7$ yr [mean \pm standard deviation]). All subjects already had some experience with the experimental setup and vibrotactile stimulation for at least $30 \mathrm{~min}$; they all took part in an earlier experiment in which the same setup was used. In the 
previous study, we evaluated the performance with vibrotactile and electrotactile feedback applied in varying orientation settings [21]. Typically, the time between practice and the previous experiment was between 0.5 and 4 weeks, except for one subject who performed a training session of $30 \mathrm{~min}$ before the start of the current experiment.

\section{Vibrotactile Feedback}

Vibrotactile feedback about the hand opening was given by an array of eight small commercially available coin motors (iNEED (HK) Limited; Shenzhen, China). We chose vibrotactile feedback because it is easy to apply, nondisturbing to the environment, and noninvasive [11]. Furthermore, we chose the coin motors because of their small size and low cost. The coin motors already showed positive results in a study on force feedback by Pylatiuk et al. [22]. We attached the vibrotactile stimulators to the forearm of the subjects using double-sided adhesive rings (EEG Kleberinge, GVB-geliMED KG; Bad Segeberg, Germany). The stimulators were controlled by a custom-made current stimulator on batteries, and their separate activation was controlled with a LabVIEW application (National Instruments; Austin, Texas) built for this purpose. The driving current of the stimulators was primarily set to $44 \mathrm{~mA}$ for each stimulator, which resulted in clearly tangible, but comfortable, sensations. We checked perceived stimulation strengths and adjusted the stimulation amplitudes if necessary to create equally perceived amplitudes of stimulation for each stimulator. The stimulators were equally distributed over the dorsal side of the forearm between the wrist and elbow (Figure 1). The resulting distance between the center of the stimulators was $3.0 \pm 0.2 \mathrm{~cm}$. For each hand position, we activated one single stimulator. Activation of the first stimulator (near the elbow) corresponded with a completely opened hand and activation of the last stimulator (at the wrist) corresponded with a fully closed hand. Activation of the intermediate stimulators was equally distributed over the range of hand movements.

\section{Virtual Environment}

In LabVIEW, we created a virtual representation of a closing and opening hand (Figure 2). The hand opening was controlled by using the scroll wheel of an adjusted computer mouse. We removed the clicks normally felt during scrolling and randomized the gain between the scrolling and the resulting hand opening to avoid information

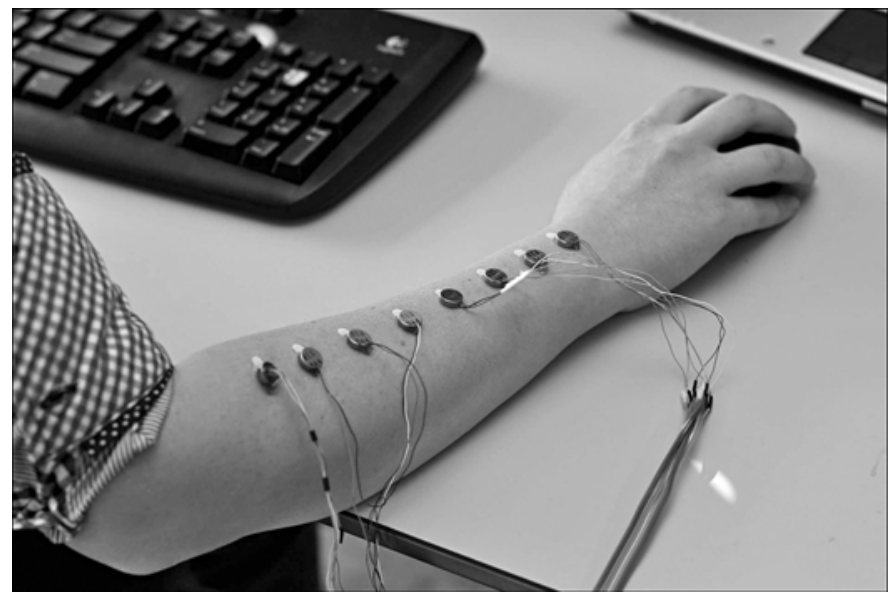

Figure 1.

Experimental setup. Eight coin motors (iNEED (HK) Limited; Shenzhen, China) placed in array on forearm.
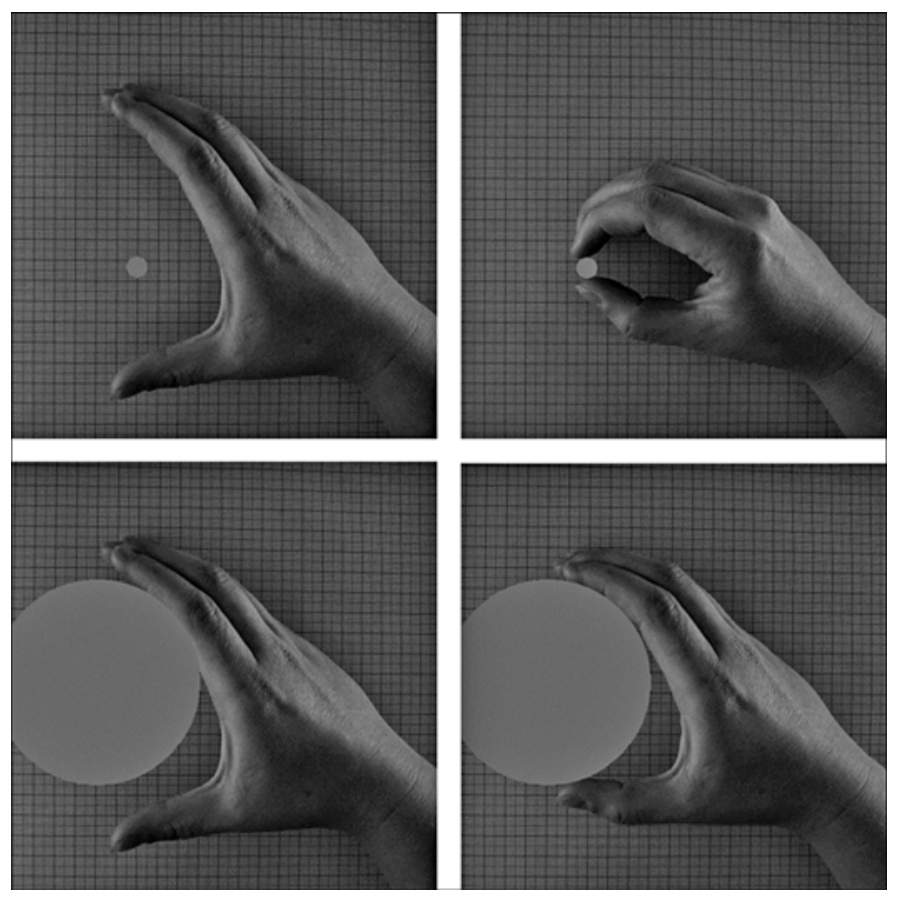

Figure 2.

Virtual representation of opening and closing hand grasping smallest and largest possible virtual objects.

coming from the mouse movement about the hand opening. Eight virtual objects (filled circles) with sizes corresponding to the eight hand positions, between minimal and maximal hand opening, were also simulated on the screen. A series of 45 randomly selected objects comprised one 
task for each experimental condition. At the start of a task, an object was shown for $0.5 \mathrm{~s}$, then it disappeared. We instructed the subjects to open or close the hand to grasp the displayed object by scrolling the mouse and hold the hand in the position that they perceived as the correct position for $2 \mathrm{~s}$. After these $2 \mathrm{~s}$, another object was displayed.

\section{Experimental Procedure}

We sat the subjects comfortably in a quiet room, behind a laptop computer screen showing the virtual environment. Subjects controlled the computer mouse with the arm they usually used to control a computer mouse, and we placed the stimulators on the same arm. The subjects wore headphones to block the auditory cues about the position of the stimulator being activated and to present the auditory stimuli used in the secondary task.

We set up a double-task procedure to evaluate the effect of distraction on the performance in a grasping task while feedback was provided. The primary task was the grasping task, performed with and without vibrotactile feedback about the hand opening. In both cases, the moving hand was not visible and therefore no visual feedback could be used. The secondary task was an auditory counting task. We chose an auditory task to avoid interference of the secondary task with the primary task. Beeps with low, middle, and high tones were presented randomly and within random time intervals to the subjects while they performed the grasping tasks. We created three different levels of distraction. For the lowest level of distraction (level 1), the beeps were presented without requiring any action by the subject. For the second level of distraction, middle and high beeps were presented and the subject had to count the number of high beeps. After completing the task, the subject reported the total number of high beeps. The third level of distraction was achieved by presenting low, middle, and high beeps and asking the subject to continuously add the number of high beeps and subtract the number of low beeps. Subjects performed every secondary task twice, with and without the help of vibrotactile feedback during the grasping task. In both conditions, visual feedback was not available, forcing the subjects to fully rely on the feedback. Subjects performed five complete training tasks with the moving hand visible on the computer screen to get used to the distraction and the feedback before the real experiment. The number of objects and the prescribed goals of these tasks were the same as those for the experimental tasks. The first five objects in every experimental condition were also used for extra training and to get used to the change in experimental conditions. We did not incorporate data from these objects in further analysis. Table 1 shows a summary of the experimental conditions.

Tasks 6 to 13 were randomized over the subjects to cancel out the effect of training on the performance in the grasping tasks. After each task, we asked the subject to indicate the perceived difficulty of the task on a visualanalog scale (VAS). The VAS, ranging from "very easy" to "very difficult," was shown on the screen and the subject placed the cursor at the perceived difficulty.

\section{Data Analysis}

The outcome parameters of the experiments were the (1) duration of the task, (2) percentage of correct hand positions, (3) mean absolute position error, and (4) percentage of achieved hand positions with a deviation up to one position. These parameters are all indicators of the performance of the subject in a grasping task. We also used the number of mistakes made in the auditory task as an outcome measure to check whether the subject adequately performed the secondary task.

For all four outcome measures, we evaluated differences over the experimental conditions with a repeatedmeasures analysis of variance (ANOVA). The factors in this analysis were the feedback condition (vibrotactile or no feedback) and the distraction level. Specific differences were post-hoc evaluated on significance by separate Student $t$-tests. Bonferroni correction on the significance level was applied to correct for the number of repeated tests.

Table 1.

Experimental conditions.

\begin{tabular}{rlcl}
\hline Task & \multicolumn{1}{c}{ Feedback } & $\begin{array}{c}\text { Distraction } \\
\text { (level) }\end{array}$ & Comment \\
\hline 1 & No primary task & 2 & Training \\
2 & No primary task & 3 & Training \\
3 & Visual & - & Training \\
4 & Visual and vibrotactile & - & Training \\
5 & Vibrotactile & - & Training \\
6 & Vibrotactile & 1 & Experiment \\
7 & Vibrotactile & 2 & Experiment \\
8 & Vibrotactile & 3 & Experiment \\
9 & Vibrotactile & - & Experiment \\
10 & - & - & Experiment \\
11 & - & 1 & Experiment \\
12 & - & 2 & Experiment \\
13 & - & 3 & Experiment \\
\hline \hline
\end{tabular}




\section{RESULTS}

We calculated percentages of correct hand positions for each task from the desired and actual hand positions. Figure 3(a) shows the distribution of these values over all subjects, categorized for the different levels of distraction and grouped for both vibrotactile feedback conditions. Figure 3(b) and 3(c) show the distribution of the percentages of hand positions with deviations up to one position and the mean absolute error. The duration of the tasks, without the $2 \mathrm{~s}$ of stable grasping per object, is shown per distraction level and feedback type in Figure 3(d). (a)

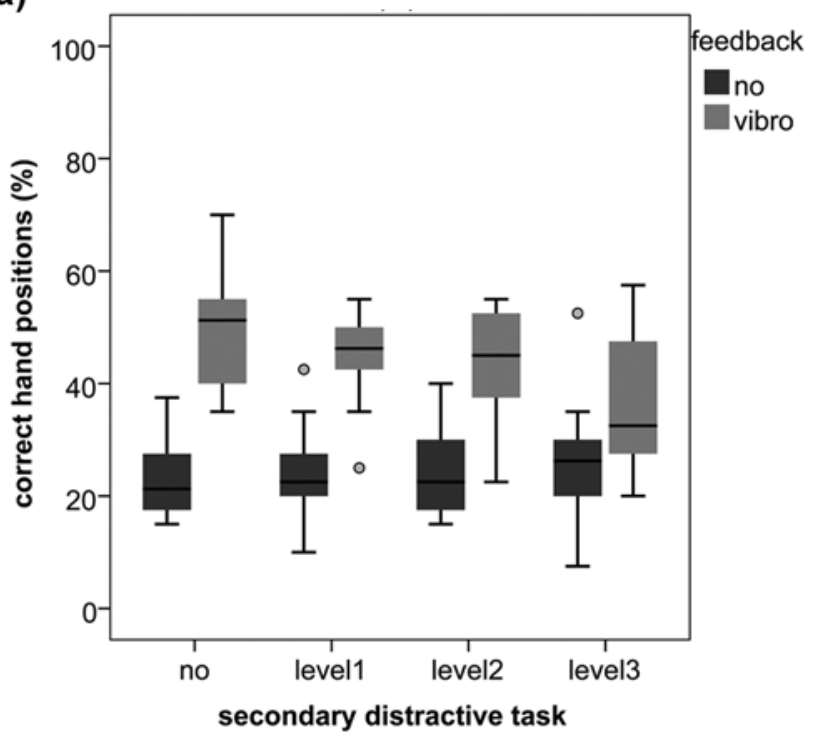

(c)

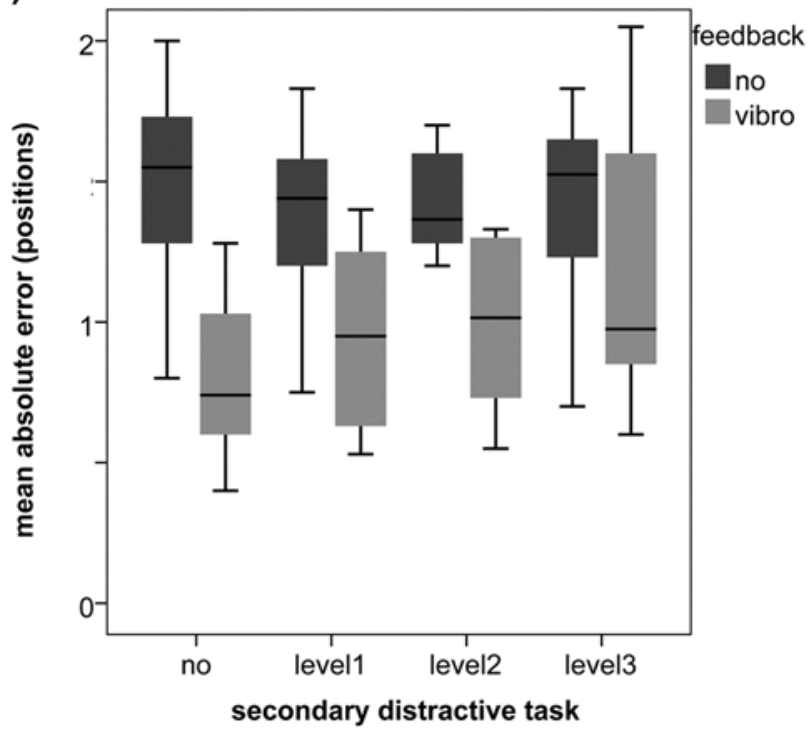

(b)

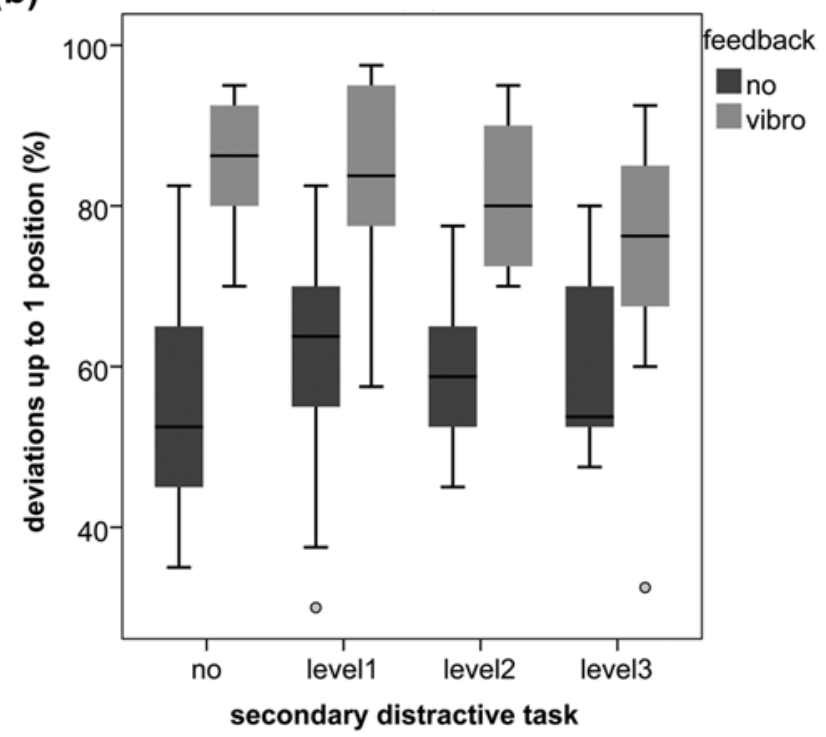

(d)

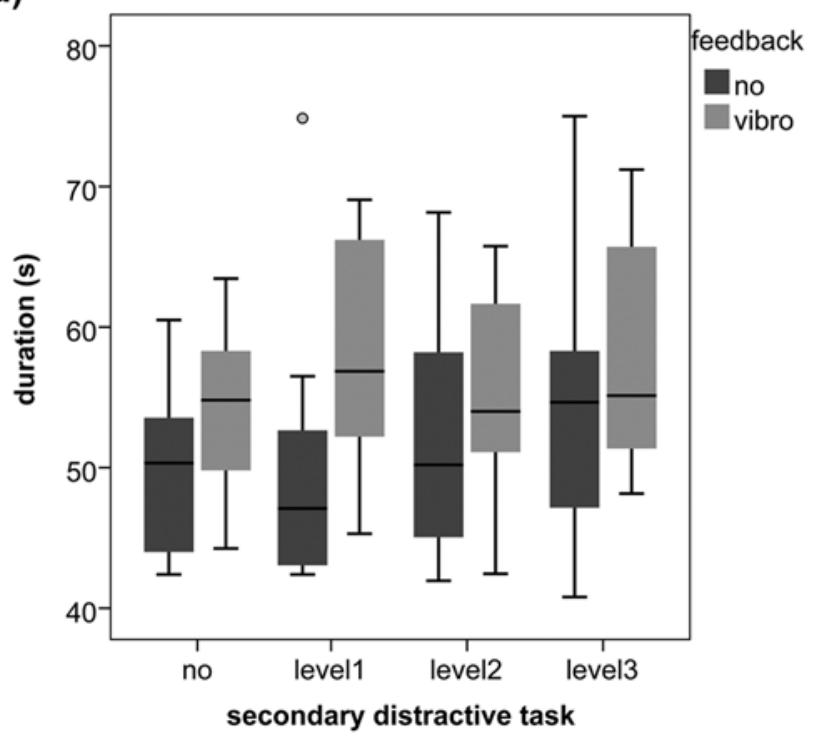

Figure 3.

Distribution of (a) percentages of correct hand positions, (b) percentages of deviations up to one position, (c) mean absolute error, and (d) duration of tasks per distraction level and feedback type (Table 1). Horizontal bars represent median value, outer sides of boxes represent 25 and 75 percentiles, and whiskers represent minimum and maximum values. Small circles represent outliers with values larger than 1.5 times interquartile range from box edge. Vibro $=$ vibrotactile. 
We analyzed the effects of the vibrotactile feedback and the distractive secondary task on the four outcome parameters with repeated-measures ANOVA. The corresponding ANOVA tables are shown for all parameters, and significance is indicated at the 95 percent level (Table 2).

Feedback shows a significant effect on all four performance parameters. The addition of vibrotactile feedback increases the percentage of correct hand positions and the percentage of hand positions with a deviation up to one position. The mean absolute error between correct and derived hand positions is significantly decreased. However, the addition of feedback also significantly increases the time needed to complete the task. The duration of the tasks was also significantly influenced by distraction, but we saw no effect of distraction for the other performance parameters. However, for the percentage of correct hand positions, we found a marginally significant interaction for effect of feedback and distraction. To evaluate this interaction, we evaluated the effects of distraction for both feedback conditions separately.

Distraction was shown to have a significant effect $(p=0.01)$ on the percentage of correct hand positions only in the vibrotactile feedback conditions ( $p=0.81$ for

Table 2.

Analysis of variance (ANOVA) for all four outcome parameters with feedback and distractions as factors.

\begin{tabular}{lccc}
\hline \multicolumn{1}{c}{ ANOVA } & $\boldsymbol{d} \boldsymbol{f}$ & $\mathbf{F}$ & $\boldsymbol{p}$-Value \\
\hline Percentage of Correct Hand Positions & & \\
Feedback & 1 & 34.09 & $0.00^{*}$ \\
Distraction & 3 & 1.80 & 0.17 \\
Feedback•Distraction & 3 & 3.06 & $0.05^{*}$ \\
Percentage of Deviations Up to One Position & \\
Feedback & 1 & 75.83 & $0.00^{*}$ \\
Distraction & 3 & 0.88 & 0.46 \\
Feedback•Distraction & 3 & 1.69 & 0.19 \\
Mean of Absolute Error in Positions & & \\
Feedback & 1 & 36.59 & $0.00^{*}$ \\
Distraction & 3 & 0.83 & 0.49 \\
Feedback•Distraction & 3 & 2.87 & 0.06 \\
Duration & & & \\
Feedback & 1 & 14.92 & $0.01^{*}$ \\
Distraction & 3 & 3.00 & $0.05^{*}$ \\
Feedback•Distraction & 3 & 1.36 & 0.28 \\
\hline
\end{tabular}

${ }^{*}$ Significance level $=0.05$.

$d f=$ degrees of freedom, $\mathrm{F}$ = between-group/within-group variability. the nonfeedback conditions). We compared performances at a given level of distraction with the experimental conditions without any distraction and evaluated them by post-hoc analysis. The $p$-values were $0.18,0.08$, and 0.01 , respectively, for the three levels at increasing difficulty, so only a significant influence of distraction on the percentage of correct hand positions was shown for the secondary task with the highest difficulty compared with the performance in tasks without distraction.

Figure 4 shows the perceived difficulty for the different levels of distraction and type of feedback provided. A large range for the perceived difficulty for the nonfeedback condition when no secondary task was used can be seen. Almost the whole range of the VAS is used for this specific condition, showing a large variation in perceived difficulty over the subjects. We analyzed the influence of the distractive secondary tasks and the provided feedback on the perceived difficulty via repeated-measures ANOVA (Table 3).

We saw a significant influence for both the type of feedback provided and the secondary distraction tasks. When no feedback was given through vibrotactile stimulation, subjects perceived the tasks to be more difficult

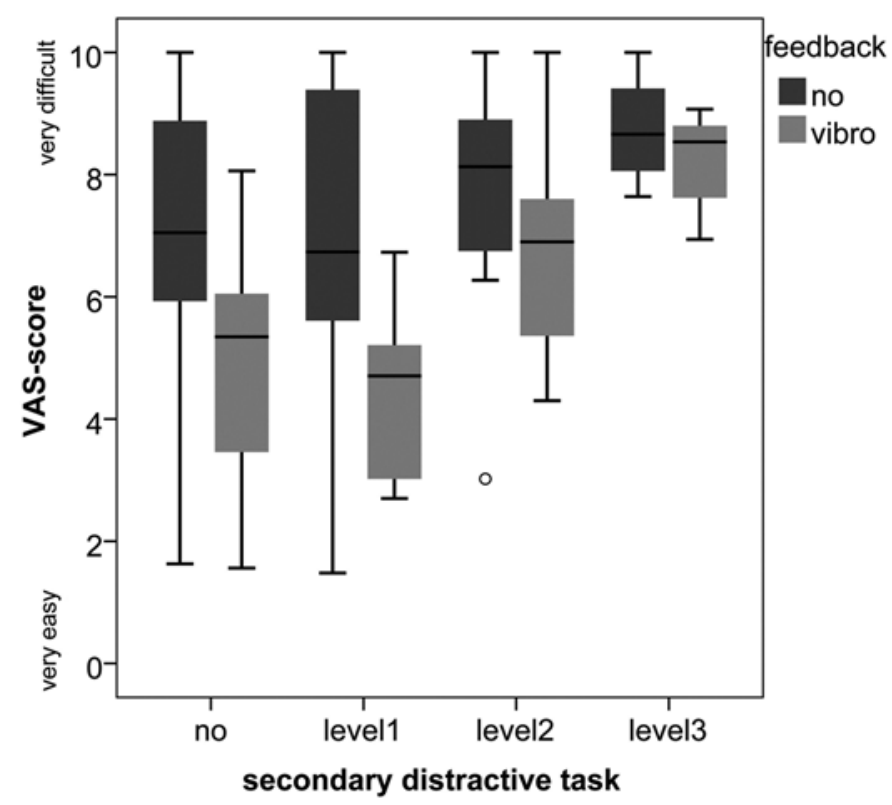

Figure 4.

Distribution of visual analog scale (VAS) scores for perceived difficulty of tasks per distraction level and feedback type. Horizontal bars represent median value, outer sides of boxes represent 25 and 75 percentiles, and whiskers represent minimum and maximum values. Small circles represent outliers with values larger than 1.5 times interquartile range. Vibro = vibrotactile. 
Table 3.

Analysis of variance (ANOVA) table for comparison of visual analog scale scores for perceived difficulty. Factors in analysis were feedback type and distraction.

\begin{tabular}{lcrc}
\hline \multicolumn{1}{c}{ ANOVA } & $\boldsymbol{d} \boldsymbol{f}$ & \multicolumn{1}{c}{ F } & $\boldsymbol{p}$-Value \\
\hline Feedback & 1 & 6.19 & $0.04^{*}$ \\
Distraction & 3 & 13.53 & $0.00^{*}$ \\
Feedback•Distraction & 3 & 1.94 & 0.15 \\
\hline
\end{tabular}

${ }^{*}$ Significance level $=0.05$.

$d f=$ degrees of freedom, $\mathrm{F}=$ between-group/within-group variability.

than in the feedback condition. Feedback helped the subjects perform the grasping task. To evaluate the effects of the different levels of distraction on the perceived difficulty, we performed post-hoc pairwise comparison of the VAS scores by Student $t$-tests. We performed six pairwise tests for each feedback condition and therefore used Bonferroni correction for multiple comparisons. We found no differences in VAS scores for the nonfeedback conditions; all tasks were perceived to be equally difficult. For the vibrotactile feedback conditions, subjects perceived that tasks performed with level 2 or 3 distraction were more difficult than tasks without distraction $(p=0.01$ and $p<$ 0.001 , respectively), while we found no significant difference in perceived difficulty between experimental conditions with no distraction and with level 1 distraction.

\section{DISCUSSION}

The number of publications on sensory feedback for users of myoelectric forearm prostheses has been increasing over the last few years, confirming the need for these applications. However, the focus is mostly on force feedback, while hand-opening feedback is also required. In this study, we describe a new approach using an array of eight vibrotactile stimulators that has been shown to increase the performance in a grasping task. Furthermore, this study provides an evaluation of the usability of the feedback in real-life situations by investigating the influence of secondary tasks on the performance.

\section{Effect of Vibrotactile Feedback}

We compared the performance of the subjects in the grasping tasks with the help of vibrotactile feedback and with the nonfeedback situations, expressed in the four outcome parameters. The results of this study showed that feedback about the hand opening through vibrotac- tile stimulation on the forearm significantly increased the percentage of correct hand positions and reduced the mean deviation from the correct hand position. However, the addition of feedback also significantly increased the time needed to complete the grasping tasks. These results correspond with the results of Kuchenbecker et al., Stepp and Matsuoka, and Wheeler et al., who also showed increases in their performance parameters at the cost of an increase in duration of the tasks $[14,20,23]$. Note that the feedback modalities and stimulation methods they used are not comparable with those used in this study.

The increase in duration found in this study was $4.5 \mathrm{~s}$ on average for the whole task of grasping 40 objects, which is $0.11 \mathrm{~s}$ per object. It can be questioned and should be further investigated whether this increase really impedes the grasping movement.

We also evaluated subjective difficulty ratings for the experimental conditions with vibrotactile feedback and the nonfeedback conditions. Tasks in which no feedback was provided (also no visual feedback) were perceived significantly more difficult than tasks in which subjects received the hand opening feedback by vibrotactile stimulation. These results also coincide with the results of Stepp and Matsuoka [20]. However, the spread in difficulty ratings was extremely large for the nonfeedback conditions. A subset of the subjects rated the nonfeedback experimental conditions as difficult, probably because they felt uncertain about their performance, while the other subjects perceived the task as easy because they did not have to pay attention to the feedback and there were no consequences for poor performance.

\section{Effect of Distraction and Levels of Distraction Applied}

Evaluation of the difficulty ratings showed a clear increase in perceived difficulty when the difficulty level was increased. Three levels of distraction (none and levels 2 and 3) could be separated from each other by differences in perceived difficulty. We found no differences in perceived difficulty between the experimental conditions without any auditory stimuli and conditions in which the auditory stimuli had to be ignored by the subjects. This showed that the auditory stimuli could indeed be ignored and did not interfere with the cognitive primary task. Every distraction is therefore related to the counting tasks and not to the presence of auditory stimuli. The previously mentioned results were only applicable for the vibrotactile feedback conditions. In the nonfeedback conditions, we 
found no significant differences in the perceived difficulty for the different distraction tasks. This is likely due to the extremely large spread in perceived difficulty over the subjects.

A trend in decreasing performance with increasing difficulty of the secondary tasks was shown in Figure 3. However, the percentage of correct hand positions significantly worsens only for the level 3 distraction compared with the tasks performed without any secondary tasks. The other performance parameters were not significantly influenced by the secondary tasks. This is largely comparable with the results found by Stepp and Matsuoka, who showed an increase in trial times but no significant decrease in performance [20]. In their study, they used only one level of a distractive cognitive secondary task and force feedback method through one stimulator, which cannot be compared with the tasks used in our experiment. The increase in trial times is also shown in our results, indicating that the secondary tasks were attention-demanding. In our experiment, a resting period was incorporated in the tasks, during which the hand had to be kept in the perceived correct position for $2 \mathrm{~s}$. Auditory stimuli were presented randomly over the whole duration of the task and therefore also occurred during the resting period, which did not influence the duration of the task. The influence of distraction on the task duration, therefore, can be even larger.

\section{Methodological Considerations}

We used a virtual environment to simulate the opening and closing of a (prosthetic) hand because this enabled us to use nondisabled subjects instead of subjects from the small pool of patients with amputation. However, the results of this study should also be affirmed for this group of potential users. The normal pathways of hand-opening feedback in nondisabled subjects are blocked by the virtualization and the only feedback available is the visual and/or the vibrotactile feedback, which is highly comparable with the situation of a myoelectric prosthesis. The movement of the virtual hand is controlled by the scroll wheel of a computer mouse and therefore not comparable with the myoelectric control of today's prostheses. We did not use myoelectric control in this experiment because a long period of training is required and the differences between subjects in the ability to control the hand by electromyography were large, as was shown in preliminary experiments. The muscles used to control the scroll wheel are, to some extent, com- parable with those used to control the hand opening, and therefore the most intuitive way to control the virtual hand without a direct relation to the hand opening. To remove clues providing information about the hand opening other than the visual and vibrotactile ones, we removed the click mechanism from the mouse scroll wheel and randomized the gain between the scroll wheel movement and the hand movement. This approach has shown to be effective because the performance in the nonfeedback experimental conditions was comparable with the results that can be expected for pure guessing. Scores of 16 percent were expected, but the scores were somewhat higher (20\%-25\%) in our study because of the known end positions of the hand.

We chose the secondary auditory counting task to prevent structural interference with the primary task. However, a secondary task involving the measurement of reaction times is a somewhat more commonly used and the accepted method [17-18].

The VAS used to evaluate the perceived difficulty is a generally used method to evaluate subjective scores. However, some problems were encountered in our experiments for the most difficult tasks. When a task was perceived as difficult, this was scored at the end of the scale, but another even more difficult task could not be scored higher on this scale. This resulted in difficulty scores clustered around the end point of the VAS. This phenomenon has been described more often in studies where VAS scores are used [24]. A VAS scale without fixed end points could have solved this problem [25] and probably more differences between VAS scores could be seen.

\section{Recommendations and Practical Implications}

The effect of training on the performance in the grasping tasks when feedback is provided should not be underestimated. Based on a recent study on the effect of training of tasks with vibrotactile feedback of force, we expect that the performance in grasping tasks will improve significantly with training [26]. Furthermore, research on motor learning has demonstrated that the duration of tasks decreases and automation of task execution improves after training [27]. This means that feedback will be processed at a more subconscious level and, therefore, secondary tasks can be expected to have even less influence. While these aspects play an important role, it is most important to develop a method that requires the least amount of attention at the first use to avoid early rejection of the prosthesis. 


\section{CONCLUSIONS}

Although distraction influences the performance in a grasping task when feedback is provided, these effects were not significant for the first two levels of distraction. It can be concluded that hand-opening feedback through an array of vibrotactile stimulators is successful because it significantly improves the performance in grasping tasks while requiring minimal attention. However, the practical implications of this study for prosthesis users cannot be fully derived from this study and should be further investigated.

\section{ACKNOWLEDGMENTS}

\section{Author Contributions:}

Study concept and design: H. J. B. Witteveen, L. de Rond. Acquisition of data: L. de Rond.

Analysis and interpretation of data: L. de Rond, H. J. B. Witteveen. Drafting of manuscript: H. J. B. Witteveen.

Critical revision of manuscript for important intellectual content: J. S. Rietman, P. H. Veltink.

Statistical analysis: L. de Rond, H. J. B. Witteveen.

Financial Disclosures: The authors have declared that no competing interests exist.

Funding/Support: This material was based on work supported by the Dutch Ministry of Economic Affairs and the Province of Overijssel, within the Pieken in de Delta initiative, the Netherlands (grant PID082035/1.6.1b). All authors are affiliated with the MIRA Institute for Biomedical Technology and Technical Medicine, University of Twente, Enschede, the Netherlands

Additional Contributions: The authors would like to thank Ed Droog for the development and realization of the measurement equipment. Institutional Review: The study protocol was approved by the local medical ethical committee (Medisch Ethische Toetsings Commissie Twente), and all subjects signed informed consent prior to the experiments.

Participant Follow-Up: The authors do not plan to inform participants of the publication of this study.

\section{REFERENCES}

1. Phillips CA. Sensory feedback control of upper- and lowerextremity motor prostheses. Crit Rev Biomed Eng. 1988; 16(2):105-40. [PMID:3053043]

2. Plettenburg DH, Herder JL. Voluntary closing: A promising opening in hand prosthetics. Technol Disabil. 2003; 15:85-94.

3. Szeto AY. Comparison of codes for sensory feedback using electrocutaneous tracking. Ann Biomed Eng. 1977;5(4):
367-83. [PMID:607824]

http://dx.doi.org/10.1007/BF02367316

4. Pons JL, Ceres R, Rocon E, Reynaerts D, Saro B, Levin S, Van Moorleghem W. Objectives and technological approach to the development of the multifunctional MANUS upper limb prosthesis. Robotica. 2005;23(3):301-10. http://dx.doi.org/10.1017/S0263574704001328

5. Atkins DJ, Heard DC, Donovan W. Epidemiologic overview of individuals with upper-limb loss and their reported research priorities. J Prosthet Orthot. 1996;8(1):2-11.

6. Peerdeman B, Boere D, Witteveen H, in 't Veld RH, Hermens H, Stramigioli S, Rietman H, Veltink P, Misra S. Myoelectric forearm prostheses: state of the art from a user-centered perspective. J Rehabil Res Dev. 2011;48(6): 719-37. [PMID:21938658] http://dx.doi.org/10.1682/JRRD.2010.08.0161

7. Mann RW, Reimers SD. Kinesthetic sensing for EMG controlled Boston arm. IEEE Trans Man Mach Syst. 1970;11: 110-15. http://dx.doi.org/10.1109/TMMS.1970.299971

8. Alles DS. Information transmission by phantom sensations. IEEE Trans Man Mach Syst. 1970;11(1):85-91. http://dx.doi.org/10.1109/TMMS.1970.299967

9. Dhillon GS, Horch KW. Direct neural sensory feedback and control of a prosthetic arm. IEEE Trans Neural Syst Rehabil Eng. 2005;13(4):468-72. [PMID:16425828] http://dx.doi.org/10.1109/TNSRE.2005.856072

10. Prior RE, Lyman J. Electrocutaneous feedback for artificial limbs. Summary progress report. February 1, 1974, through July 31, 1975. Bull Prosthet Res. 1975;(10-24):3-37. [PMID:1227686]

11. Kaczmarek KA, Webster JG, Bach-y-Rita P, Tompkins WJ. Electrotactile and vibrotactile displays for sensory substitution systems. IEEE Trans Biomed Eng. 1991;38(1):1-16. [PMID:2026426] http://dx.doi.org/10.1109/10.68204

12. Jones LA, Sarter NB. Tactile displays: guidance for their design and application. Hum Factors. 2008;50(1):90-111. [PMID:18354974] http://dx.doi.org/10.1518/001872008X250638

13. Blank A, Okamura AM, Kuchenbecker KJ. Effects of proprioceptive motion feedback on sighted and non-sighted control of a virtual hand prosthesis. Proceedings of the Symposium on Haptic Interfaces for Virtual Environment and Teleoperator Systems; 2008 Mar 13-14; Reno, NV.

14. Kuchenbecker KJ, Gurari N, Okamura AM. Effects of visual and proprioceptive motion feedback on human control of targeted movement. Proceedings of the IEEE 10th International Conference on Rehabilitation Robotics; 2007 Jun 13-15; Noordwijk, the Netherlands.

15. Asghari Oskoei M, Hu H. Myoelectric control systems-a survey. Biomed Signal Process Control. 2007;2(4):275-94. http://dx.doi.org/10.1016/j.bspc.2007.07.009 
16. Cipriani C, Zaccone F, Micera S, Carrozza MC. On the shared control of an EMG-controlled prosthetic hand: analysis of user-prosthesis interaction. IEEE Trans Robot. 2008; 24(1):170-84. http://dx.doi.org/10.1109/TRO.2007.910708

17. Huang HJ, Mercer VS. Dual-task methodology: applications in studies of cognitive and motor performance in adults and children. Pediatr Phys Ther. 2001;13(3):133-40. [PMID:17053670] http://dx.doi.org/10.1097/00001577-200110000-00005

18. Wright DL, Kemp TL. The dual-task methodology and assessing the attentional demands of ambulation with walking devices. Phys Ther. 1992;72(4):306-15. [PMID:1584862]

19. Schmidt RA. Motor control and learning: A behavioral emphasis. 2nd ed. Champaign (IL): Human Kinetics; 1988.

20. Stepp CE, Matsuoka Y. Relative to direct haptic feedback, remote vibrotactile feedback improves but slows object manipulation. Proceedings of the 2010 Annual International Conference of the IEEE Engineering in Medicine and Biology Society; 2010 Aug 31-Sep 4; Buenos Aires, Argentina.

21. Witteveen HJ, Droog EA, Rietman JS, Veltink PH. Vibroand electrotactile user feedback on hand opening for myoelectric forearm prostheses. IEEE Trans Biomed Eng. 2012; 59(8):2219-26. [PMID:22645262] http://dx.doi.org/10.1109/TBME.2012.2200678

22. Pylatiuk C, Kargov A, Schulz S. Design and evaluation of a low-cost force feedback system for myoelectric prosthetic hands. J Prosthet Orthot. 2006;18(2):57-61. http://dx.doi.org/10.1097/00008526-200604000-00007

23. Wheeler J, Bark K, Savall J, Cutkosky M. Investigation of rotational skin stretch for proprioceptive feedback with application to myoelectric systems. IEEE Trans Neural
Syst Rehabil Eng. 2010;18(1):58-66. [PMID:20071271]

http://dx.doi.org/10.1109/TNSRE.2009.2039602

24. Langley GB, Sheppeard H. The visual analogue scale: its use in pain measurement. Rheumatol Int. 1985;5(4):145-48. [PMID:4048757] http://dx.doi.org/10.1007/BF00541514

25. Gescheider GA. Psychophysics: Method, theory, and application. 2nd ed. Hillsdale (NJ): L. Erlbaum Associates; 1985.

26. Stepp CE, Chang C, Malhotra M, Matsuoka Y. Vibrotactile feedback aids EMG control of object manipulation. Proceedings of the 2011 Annual International Conference of the IEEE Engineering in Medicine and Biology Society; 2011 Aug 30-Sep 3; Boston, MA.

27. Halsband U, Lange RK. Motor learning in man: a review of functional and clinical studies. J Physiol Paris. 2006;99(4-6): 414-24. [PMID:16730432] http://dx.doi.org/10.1016/j.jphysparis.2006.03.007

Submitted for publication December 21, 2011. Accepted in revised form April 19, 2012.

This article and any supplementary material should be cited as follows:

Witteveen HJ, de Rond L, Rietman JS, Veltink PH. Handopening feedback for myoelectric forearm prostheses: Performance in virtual grasping tasks influenced by different levels of distraction. J Rehabil Res Dev. 2012; 49(10):1517-26.

http://dx.doi.org/10.1682/JRRD.2011.12.0243

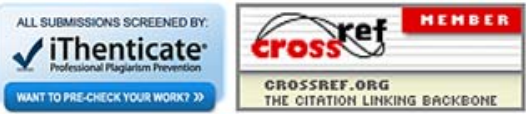

